\title{
Relação entre variabilidade da frequência cardíaca e aumento no desempenho físico em jogadores de futebol
}

\section{The correlation between heart rate variability and improvement in soccer player's physical performance}

\author{
Ricardo Santos Oliveira ${ }^{1}$ \\ Rafael Evangelista Pedro \\ Vinícius Flávio Milanez \\ Henrique Bortolotti ${ }^{1}$ \\ Marcelo Vitor-Costa ${ }^{1}$ \\ Fábio Yuzo Nakamura
}

Resumo - O objetivo deste estudo foi analisar se a variabilidade da frequência cardíaca (VFC) analisada no período inicial da pré-temporada de futebol apresenta relação com a melhora do desempenho físico ao longo deste período. Dez jogadores de futebol fizeram parte da amostra. O desempenho foi analisado através dos seguintes testes: Yo-Yo Intermittent Recovery Test Level 1 (Yo-Yo IR1), sprint de $30 \mathrm{~m}$ e salto vertical, aplicados antes e após seis semanas da pré-temporada. As medidas de VFC foram realizadas em repouso, na posição supina, durante dez minutos. O teste de Spearman foi usado para investigar as possíveis relações entre VFC e melhora no desempenho e o teste T de Student para verificar as mudanças no desempenho e na VFC. A inferência prática baseada em magnitudes (analise qualitativa) foi aplicada para verificar as chances dos valores encontrados serem positivos, irrelevantes e negativos. Houve melhora significante do desempenho para o Yo-Yo IR1 $(P<0,001)$ e Sprint de $30 \mathrm{~m}(P<0,001)$. A análise qualitativa revelou que a mudança do desempenho no Yo-Yo IR1 foi "muito provavelmente positiva", para o Sprint de $30 \mathrm{~m}$ foi "quase certamente positiva" e para o salto vertical foi "inconclusiva". Houve forte correlação entre um índice parassimpático da VFC e a variação no desempenho [r $=0,85 ; \mathrm{P}=0,003$ (IC95\% =0,49 - 0,97)]. Em conclusão, esse estudo mostrou uma correlação forte entre índices parassimpáticos da VFC analisados antes do treinamento, com a melhora do desempenho no Yo-Yo IR1 durante a pré-temporada em atletas de futebol. Palavras-chave: Atletas; Esporte; Fisiologia cardiovascular; Testes de aptidão.

Abstract - The aim of this study was to analyze whether the heart rate variability (HRV), assessed at the beginning of a soccer preseason, reveals a correlation with the improvement of physical performance over this training period. Ten soccer players took part in the study. Their performance was evaluated by the following tests: the Yo-Yo intermittent recovery test, Level 1(Yo-YoIR1); the 30-m sprint time, and the vertical jump; all were conducted before and after six weeks of pre-season. The HRV measurements were performed at rest in the supine position for ten minutes. The Spearman Rank Correlation test was used to investigate any possible relationship between HRV and improvements in performance, while the Student's $t$-test verified the changes in both performance and HRV. The Magnitude-Based Inferences approach (qualitative analysis) was applied to verify the possibilities of the observed values being positive, negative or inconclusive. There were significant improvements in Yo-Yo IR1 performance $(P<0.001)$ and in the $30-m$ sprint time $(P<0.001)$. The qualitative analysis revealed that the differences in Yo-Yo IR1 performance were very likely positive, were almost certainly positive for the sprint, but were inconclusive for the vertical jump. There was a strong correlation between one parasympathetic index and the change in performance $[r=$ $0.85 P=0.003($ IC95\% $=0.49-0.97)]$. In conclusion, this study revealed a strong correlation between parasympathetic indices of HRV (analyzed before the training) with the performance improvement in Yo-Yo IR1 in football athletes during pre-season.

Key words: Athletes; Cardiovascular physiology; Physical fitness; Sport.
1 Universidade Estadual de Londrina. Grupo de Estudo das Adaptações Fisiológicas ao Treinamento. Londrina, PR. Brasil.

Recebido em 27/04/11 Revisado em 07/06/12 Aprovado em 15/07/12 


\section{INTRODUÇÃO}

O futebol é uma modalidade esportiva acíclica caracterizada por esforços intermitentes com curtos intervalos de recuperação entre as ações, intercalando esforços de alta e baixa intensidade. Embora o metabolismo aeróbio forneça a maior proporção de energia para as atividades durante os jogos (81 a 92\%)', foi demonstrado que jogadores de elite realizam $28 \%$ mais corridas de alta intensidade e $58 \%$ mais sprints em comparação a jogadores profissionais de nível inferior ${ }^{2}$. Consequentemente, capacidades tais como a força, velocidade e resistência aeróbia/anaeróbia são imprescindíveis para a prática do futebol em alto nível. Dessa forma, o período de preparação física na pré-temporada dos atletas deve ser direcionado para aprimorar tais capacidades, em decorrência de adaptações fisiológicas almejadas e, consequentemente, melhora do desempenho ${ }^{3}$.

Para monitorar as melhoras no desempenho, o Yo-Yo Intermittent Recovery Test level 1 (Yo-Yo IR1) tem sido amplamente utilizado no futebol ${ }^{4}$. O desempenho neste teste apresenta alta correlação com as distâncias percorridas em alta intensidade, durante partidas de futebol ${ }^{5}$, discrimina diferentes níveis competitivos e é sensível às adaptações ao longo da pré-temporada. ${ }^{4,5}$ Os determinantes fisiológicos do desempenho no Yo-Yo IR1 são relacionados com as exigências fisiológicas durante o jogo ${ }^{6}$, as quais não dependem somente do consumo máximo de oxigênio, mas também da capacidade de manter o equilíbrio ácido-base, e de condicionantes neuromusculares?.

As adaptações fisiológicas também têm sido monitoradas por meio das respostas do sistema nervoso autônomo, analisadas a partir da variabilidade da frequência cardíaca (VFC), que pode fornecer informações úteis quanto a alterações no estado de treinamento ${ }^{8}$. Tem sido observado que a VFC é sensível a alterações nas cargas de treinamento ${ }^{9,10}$ e parece ser uma ferramenta importante para a prescrição de treinamento individualizado ${ }^{11}$.

Além disso, a VFC analisada no período prévio a um programa de treinamento apresentou relação positiva com o aumento no $\mathrm{VO}_{2}$ max após treinamento, em não atletas ${ }^{12}$, sugerindo que a VFC pode ser importante preditora do potencial de melhora da aptidão aeróbia. Desta forma, poderíamos sugerir que a VFC também poderia estar associada a melhoras no Yo-Yo IR1, pois há relação entre desempenho no Yo-Yo IR1 e $\mathrm{VO}_{2} \max ^{4}$. No entanto, desconhecemos qualquer estudo verificando associação da VFC com desempenho em testes de campo específicos para modalidades coletivas, que exigem uma combinação de capacidades neuromusculares, metabólicas e cardiovasculares relevantes para o desempenho em campo.

Dessa forma, os objetivos do presente estudo foram: a) relacionar índices vagais de VFC com a variação do desempenho no Yo-Yo IR1 do momento 1 (M1) - início da pré-temporada, para o momento 2 (M2) - final da pré-temporada, e; b) comparar os índices de desempenho de força explosiva, velocidade e índices de VFC nos dois momentos de análise, em jogadores profissionais de futebol. 
Nossa hipótese é que existe uma correlação entre índices de VFC de repouso medidos previamente ao período de treinamento, e as mudanças relativas no desempenho em teste de campo específico para o futebol. Adicionalmente, aumentos nos indicadores de desempenho de força explosiva e velocidade são esperados após a pré-temporada.

\section{PROCEDIMENTOS METODOLÓGICOS}

\section{Sujeitos}

Inicialmente, vinte jogadores de futebol profissional pertencentes à segunda divisão do campeonato estadual (PR) participaram do estudo. Entretanto, os atletas que não participaram dos dois momentos, por lesão ou por terem sido negociados para outras equipes, foram excluídos da análise final. Consequentemente, apenas dez jogadores foram incluídos no estudo (idade: $22 \pm 3$ anos; peso corporal: 76,7 $\pm 6,9 \mathrm{~kg}$; estatura: $180 \pm 7 \mathrm{~cm}$ e IMC 23,6 $\pm 1,3 \mathrm{~kg} \cdot \mathrm{m}^{2}$ ). Da amostra total, dois eram goleiros, dois eram defensores, quatro meio-campistas, um lateral e um atacante. Após receberem informações a respeito dos procedimentos do estudo, os jogadores assinaram um termo de consentimento livre e esclarecido. $\mathrm{O}$ estudo foi aprovado pelo Comitê de Ética em Pesquisa da Universidade Estadual de Londrina (CAAE - 0202.0.268.000-10).

\section{Delineamento do estudo}

Anteriormente ao início das sessões de treinamento da pré-temporada (M1), foi avaliada a VFC de repouso dos atletas. Logo em seguida, foi realizado o Yo-Yo IR1 e, $24 \mathrm{~h}$ depois, foram realizados o teste de saltos com contra-movimento e o teste de velocidade. Ao final da pré-temporada (M2), cuja duração foi de seis semanas, os atletas repetiram o protocolo de avaliação e testes no mesmo horário, para evitar as variações circadianas. Os jogadores foram instruídos a não realizarem exercícios vigorosos por um período mínimo de 48 horas precedentes aos testes, bem como manter o consumo alimentar habitual e evitar o consumo de produtos cafeinados e bebidas alcoólicas no período de testes.

\section{Treinamento}

Durante o período preparatório (pré-temporada), que teve a duração de seis semanas, os atletas realizaram sessões de treinamento em dois períodos do dia. No período da manhã, realizavam exercícios específicos para melhora dos aspectos físicos, tais como, resistência específica, resistência de força, resistência anaeróbia, potência e velocidade, enquanto que o período da tarde era reservado para os treinos técnicos e táticos.

\section{Yo-Yo Intermittent Recovery Test Level 1 (Yo-Yo IR1)}

O Yo-Yo IR1 consiste na realização de corridas vai e vem por uma distância de $20 \mathrm{~m}$, realizadas com aumento de velocidade em estágios determinados, com 10 s de recuperação ativa entre as corridas. Para isso, foi demarcado 
com cones de sinalização o trajeto a ser percorrido, bem como a área destinada à recuperação ativa. A velocidade foi controlada por sinal sonoro. O teste terminou com a exaustão voluntária ou quando o participante não alcançava por duas vezes, no mesmo estágio, as marcações (cone na extremidade inicial dos $20 \mathrm{~m}$ ). A distância total percorrida durante o teste foi considerada para a análise do desempenho ${ }^{13}$. O teste apresenta elevada reprodutibilidade, com o coeficiente de correlação intraclasse de $0,98(P<$ $0,0001)$ e coeficiente de variação de $3,5 \%$.

\section{Teste de velocidade (30m)}

O teste para avaliar a velocidade consistiu na realização de 2 sprints máximos em uma distância de 30 metros, com intervalos de $30 \mathrm{~s}$ entre os sprints. O sistema de células fotoelétricas (Multisprint", Hidrofit, Brasil) foi montado e fixado a uma altura de 1,2 metros acima do piso para registrar o tempo de cada sprint. O melhor tempo alcançado em um dos sprints foi adotado como índice de desempenho.

\section{Salto com contra movimento}

A potência de membros inferiores foi inferida a partir de salto com contra movimento. A altura do salto de cada jogador foi mensurada usando uma plataforma de salto (Multisprint, Hidrofit, Brasil). Para isolar a potência dos membros inferiores, o salto foi utilizado sem movimento dos braços. Os participantes iniciavam o teste em pé, com as mãos no quadril, e então flexionavam o quadril e joelho e imediatamente realizavam um salto o mais alto possível. Cada participante realizou três saltos, com $30 \mathrm{~s}$ de recuperação entre cada tentativa. A melhor medida foi assumida como indicador de potência. $O$ teste apresenta alta reprodutibilidade, com o coeficiente de correlação intraclasse de 0,98 e coeficiente de variação de $2,8 \%{ }^{14}$.

\section{Análise da variabilidade da frequência cardíaca (VFC)}

Os intervalos RR foram continuamente registrados durante $10 \mathrm{~min}$ em repouso, com o atleta na posição supina, por um cardiofrequencímetro (POLAR, modelo RS800 - Kempele, Finlândia). Este equipamento foi validado previamente para este tipo de análise ${ }^{15}$. Para análise da VFC, foram considerados apenas os 5 minutos finais de registro. Os batimentos ectópicos (desvio maior que $20 \%$ dos intervalos adjacentes) foram identificados e manualmente interpolados pelos intervalos R-R adjacentes.

O cálculo dos parâmetros no domínio do tempo foi realizado a partir dos índices RMSSD (raiz quadrada da média das diferenças sucessivas ao quadrado dos intervalos $\mathrm{RR}$ adjacentes), média dos intervalos $\mathrm{RR}$ ( $\mathrm{RR}$ médio) e frequência cardíaca de repouso $\left(\mathrm{FC}_{\mathrm{rep}}\right)$. A densidade do espectro de potência foi quantificada utilizando transformada rápida de Fourier, sendo os componentes de baixa frequência (LF) e de alta frequência (HF) registrados para análise. Os dados no domínio da frequência foram expressos em unidades normalizadas (un). As análises foram feitas a partir do programa Kubios HRV (University of Eastern Finland). 


\section{Analise Estatística}

Os dados são apresentados como média e desvio padrão e para a análise qualitativa os dados foram apresentados a partir da mudança relativa descrita em mudança percentual e intervalo de confiança de $90 \%{ }^{16}$. A distribuição de cada variável foi analisada com o teste de Shapiro-Wilk. Para comparação do desempenho (Yo-Yo IR1, velocidade e salto vertical) e dos índices de VFC nos momentos 1 e 2, foi utilizado o teste T de Student para amostras dependentes. Para verificar se atividade autonômica apresentava relação com o aumento no desempenho foi aplicada correlação entre a melhora no desempenho específico, analisada pela diferença percentual de M1 para M2 ( $\Delta \%$ Yo-Yo IR1) e os índices autonômicos parassimpáticos obtidos em M1. Essa análise foi realizada mediante correlação de Spearman, uma vez que o $\Delta \%$ Yo-YoIR1 não apresentou distribuição normal. A significância adotada para essas análises foi de $P<0,05$.

Em adição ao teste de hipótese nula, foi aplicada a inferência prática baseada em magnitudes ${ }^{16}$. Para isso, foi calculada a chance de um valor encontrado ser benéfico (positivo) ou prejudicial (negativo) (ex: maior ou menor que o mínimo efeito prático importante ou mínima mudança detectável [0,20 multiplicado pelo desvio padrão inicial baseado no tamanho de efeito ${ }^{17}$. Dessa forma, a mudança foi avaliada qualitativamente como se segue: $<1 \%$ quase certamente não; 1-5\% muito improvável; 5-25\% improvável; $25-75 \%$ possível; $75-95 \%$ provável; $95-99 \%$ muito provável e $>99 \%$ quase certamente sim. Se os valores negativos e positivos apresentassem resultados $>10 \%$, a inferência era considerada inconclusiva.

\section{RESULTADOS}

A tabela 1 apresenta os resultados referentes às mudanças no desempenho nos testes durante o período investigado. Houve diferença significante no desempenho no Yo-Yo IR1 $(P<0,001)$ e no teste de velocidade $(P<0,001)$. No entanto, não houve diferença no desempenho do salto vertical com contra-movimento. A análise qualitativa mostrou um aumento muito provavelmente positivo para o desempenho no Yo-Yo IR1. Da mesma forma, houve um aumento quase certamente positivo para a velocidade. Porém, não houve alterações conclusivas no desempenho para o salto vertical.

As respostas dos índices autonômicos nos momentos 1 e 2 são apresentadas na tabela 2. Nota-se que não houve diferenças significantes entre os índices. Além do teste de hipótese, a tabela apresenta também os resultados da análise qualitativa, a qual mostrou que as diferenças observadas para a $\mathrm{FC}_{\text {rep }}$ e o R-R médio foram provavelmente positivas, ao passo que para as demais variáveis a análise revelou-se inconclusiva.

A figura 1 apresenta os valores de correlação entre o RMSSD obtido em M1 e o $\Delta \%$ de variação do desempenho no Yo-Yo IR1 entre M1 e M2. Houve forte correlação entre o índice vagal e o $\Delta \%$ de variação no desempenho $\mathrm{r}=0,85 P=0,003$ (IC95\% =0,49 - 0,97). Além disso, o $\Delta \%$ de variação do desempenho no Yo-Yo IR1 apresentou forte correlação também com outros 
índices vagais $(\mathrm{HF}(\mathrm{nu})=0,60 P=0,06 \mathrm{IC95} \%=-0,05-0,89 ; \mathrm{SD} 1=0,81$ $P=0,005 \mathrm{IC}=0,37-0,95)$. No entanto, a correlação observada com o HF não apresentou significância. As correlações apresentadas entre os índices autonômicos no momento 1 e o $\Delta \%$ para a velocidade e os saltos não foram significantes $(P>0,05)$.

Tabela 1. Valores obtidos nos testes de desempenho, expressos em média (DP) em M1 e M2, e inferência prática em relação à diferença (\%). $(n=10)$

\begin{tabular}{llll}
\hline & M1 & M2 & $\mathrm{P}$ \\
\hline Yo-YoIR1 (m) & $1272(340,9)$ & $1648(458,3)$ & 0,001 \\
\% diferença (IC 90\%) & $30,1(13-47)$ & & \\
positivo/irrelevante/negativo & $98,8 / 1 / 0,2$ & & \\
Inferência qualitativa & Muito provavelmente positiva & \\
Velocidade (s) & $4,1(0,1)$ & $3,9(0,1)$ & 0,001 \\
\% diferença (IC 90\%) & $-3,7(-2,3--5,1)$ & & \\
positivo/irrelevante/negativo & $99,8 / 0,2 / 0$ & & \\
Inferência qualitativa & Quase certamente positiva & \\
\hline Salto Vertical (cm) & $45,7(4,7)$ & $45,8(7)$ & \\
\% diferença (IC 90\%) & $1(-31-33)$ & & \\
positivo/irrelevante/negativo & $45,6 / 13 / 41,3$ & & \\
Inferência qualitativa & Inconclusiva & & \\
\hline
\end{tabular}

(M1) Inicio de pré-temporada; (M2) final de pré-temporada.

Tabela 2. Valores dos índices autonômicos, média (DP) no início e fim da pré-temporada e inferência prática em relação à diferença (\%).

\begin{tabular}{|c|c|c|c|}
\hline & M1 & M2 & $P$ \\
\hline RMSSD (ms) & $90,5(56)$ & $97,9(60,3)$ & 0,58 \\
\hline \% diferença (IC 90\%) & \multicolumn{3}{|c|}{$24(-69-120)$} \\
\hline Positivo/irrelevante/negativo & \multicolumn{3}{|c|}{$53,4 / 26,4 / 20,2$} \\
\hline Inferência qualitativa & \multicolumn{3}{|c|}{ Inconclusiva } \\
\hline $\mathrm{HF}(\mathrm{nu})$ & $63,2(23,6)$ & $55(23)$ & 0,646 \\
\hline \% diferença (IC 90\%) & \multicolumn{3}{|c|}{$120(-160-390)$} \\
\hline Positivo/irrelevante/negativo & \multicolumn{3}{|c|}{$73,2 / 7,6 / 19,3$} \\
\hline Inferência qualitativa & \multicolumn{3}{|c|}{ Inconclusiva } \\
\hline LF (nu) & $36,9(23,6)$ & $45(22,9)$ & 0,646 \\
\hline \% diferença (IC 90\%) & \multicolumn{3}{|c|}{$99(-130-330)$} \\
\hline Positivo/irrelevante/negativo & \multicolumn{3}{|c|}{$72,6 / 8,8 / 18,6$} \\
\hline Inferência qualitativa & \multicolumn{3}{|c|}{ Inconclusiva } \\
\hline RRmédio (ms) & $1013,9(58,1)$ & $1083,2(110,3)$ & 0,06 \\
\hline \% diferença (IC 90\%) & \multicolumn{3}{|c|}{$6,9(1,2-13)$} \\
\hline Positivo/irrelevante/negativo & \multicolumn{3}{|c|}{$95 / 3,6 / 1,4$} \\
\hline Inferência qualitativa & \multicolumn{3}{|c|}{ Provavelmente positiva } \\
\hline FCrep (bpm) & $59,7(3,4)$ & $56,2(5,1)$ & 0,06 \\
\hline \% diferença (IC 90\%) & \multicolumn{3}{|c|}{$-5,7(-11--0,84)$} \\
\hline Positivo/irrelevante/negativo & \multicolumn{3}{|c|}{$94,5 / 3,9 / 1,6$} \\
\hline Inferência qualitativa & \multicolumn{3}{|c|}{ Provavelmente positiva } \\
\hline
\end{tabular}

(M1) inicio de pré-temporada; (M2) final de pré-temporada. RMSSD = Raiz quadrada da média dos quadrados dos intervalos RR adjacentes; $\mathrm{HF}=$ Banda de alta frequência; $\mathrm{LF}=$ Banda de baixa frequência; $\mathrm{RRmédio}=$ Intervalos RR médios; RCrep = Frequência cardíaca de repouso. 


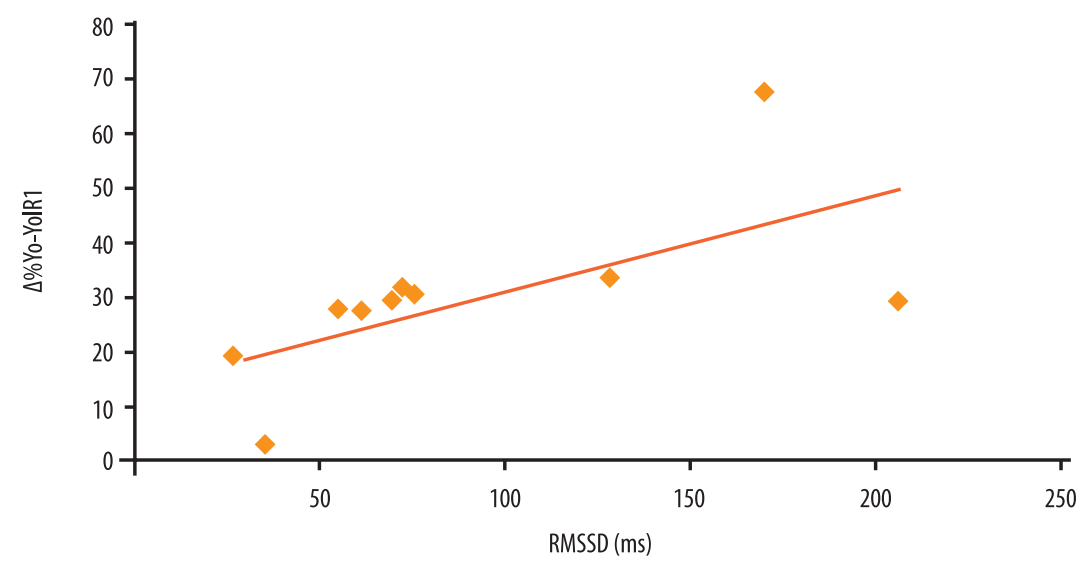

$r=0,85 p=0,003(I C=0,49-0,97)$. RMSSD $=$ Raiz quadrada da média dos quadrados dos intervalos RR adjacentes.

Figura 1. Coeficiente de correlação entre os valores de RMSSD no momento 1 e o $\Delta \%$ de variação no desempenho obtidos no momentos 1 e 2 ( $n=10)$.

\section{DISCUSSÃO}

O objetivo deste estudo foi analisar as respostas de desempenho ao longo da pré-temporada em uma equipe de futebol profissional e verificar se a VFC de repouso tem relação com a melhora no desempenho ao longo desse período.

O principal resultado do nosso estudo foi uma associação entre a VFC pré-treinamento, marcadamente dos índices mediados pelo braço parassimpático do sistema nervoso autônomo, e o $\Delta \%$ de melhora do desempenho no Yo-Yo IR1. Houve uma correlação forte e positiva entre o RMSSD, HF e SD1 e o $\Delta \%$ no Yo-Yo IR1 entre M1 e M2, na qual a variação no percentual de melhora do desempenho pôde ser explicada pela variação no RMSSD, HF e SD1, respectivamente.

Embora já tenha sido demostrada a existência de uma relação entre VFC na linha de base e melhora no $\mathrm{VO}_{2}$ max em atletas e não atletas ${ }^{12,18}$, este foi o primeiro estudo a demonstrar uma forte correlação entre os índices vagais da VFC de repouso e o $\Delta \%$ de aumento do desempenho no Yo-Yo IR1 em atletas de modalidade coletiva (Figura 1). Este teste, além de apresentar uma significativa contribuição do metabolismo aeróbio, possui também uma considerável participação do metabolismo anaeróbio ${ }^{19}$, mostrando que a VFC na linha de base parece ter relação com o aumento do desempenho também em exercícios que não dependem exclusivamente do $\mathrm{VO}_{2} \max$.

A relação encontrada entre os índices vagais da VFC e o $\Delta \%$ no desempenho corroboram os achados de Hautala et al. ${ }^{12}$ e Hedelin et al. ${ }^{18}$, os quais encontraram relação entre VFC pré-treinamento e $\Delta \%$ do $\mathrm{VO}_{2} \max$ em resposta a treinamento aeróbio. Embora tenha sido encontrada tal relação, o motivo pelo qual a VFC se relaciona com alterações no desempenho ainda é desconhecida.

Apesar da forte relação com o $\Delta \%$ do desempenho no Yo-Yo IR1, a VFC não apresentou relação com o $\Delta \%$ desempenho para a velocidade e 
potência ( $\mathrm{r}=0,28$ e 0,15 respectivamente), mostrando que a VFC parece não apresentar relação com o $\Delta \%$ no desempenho em testes que apresentam predominantemente a utilização do metabolismo anaeróbio e/ou grande participação de fatores neuromusculares.

O período de pré-temporada e início do período competitivo foi capaz de proporcionar melhoras significativas no desempenho do Yo-Yo IR1 (análise qualitativa revelando uma melhora de 30\% muito provavelmente positiva) e na velocidade, na qual se observou uma melhora significativa (-3,7\% quase certamente positiva). No entanto, não foram observadas melhoras significativas no salto vertical e nos índices de VFC (análise qualitativa revelando resultados inconclusivos).

O $\Delta \%$ do Yo-Yo IR1 (30\%) foi similar ao reportado na literatura. Bangsbo et al., ${ }^{4}$ em uma recente revisão, mostraram que existe uma significante melhora, em alguns casos de até $25 \%$, no desempenho do Yo-Yo IR1 após um período de pré-temporada. Além disso, a grande variabilidade encontrada nos nossos resultados ( $\mathrm{DP}=340,9$ no início e $458,3 \mathrm{~m}$ no fim do período analisado) pode ser explicada pelas diferentes posições dos atletas 5 .

O desempenho inicial dos atletas foi bastante inferior ao encontrado na literatura para atletas de elite ${ }^{4}$. Esta diferença pode ser explicada pelo fato de que a maioria dos atletas do presente estudo estava em período de férias antes da apresentação para a pré-temporada. No entanto, a diferença foi minimizada após o período de treinamento, pois os atletas percorreram $1648 \pm 458,3 \mathrm{~m}$, aproximando-se dos valores reportados no estudo de Krustrup et al. ${ }^{5}$, em que os atletas percorreram $1793 \pm 100 \mathrm{~m}$. Embora a diferença entre os resultados do presente estudo e os observados na literatura tenha sido minimizada durante o M2 (1648 $\pm 458,3 \mathrm{~m})$, constatamos que os atletas avaliados neste estudo são muito inferiores aos atletas de alto nível em estudos internacionais ${ }^{4}$.

Além da melhora no desempenho no Yo-Yo IR1, foram observadas melhoras na velocidade ao longo do período de treinamento; entretanto, não houve mudanças na potência de membros inferiores. Esses resultados podem indicar que a velocidade, por estar associada diretamente com o desempenho durante o jogo, é priorizada pelos preparadores físicos, ao passo que existe controvérsia acerca da importância da capacidade de salto para o desempenho em campo ${ }^{20}$.

Não foram observadas diferenças para os índices de VFC após o período de treinamento. Esses resultados podem ser explicados, em parte, devido ao fato dos índices vagais da VFC sofrerem uma saturação em atletas ${ }^{21}$, podendo ser agravada quando a medida é feita com o indivíduo na posição supina, como foi o caso do presente estudo. Em estudos futuros, outras posições corporais (por exemplo, em pé), deverão ser testadas.

Algumas limitações do presente estudo foram a falta de uma medida da carga interna de treinamento ao longo do período de preparação e medidas de FC de recuperação. Talvez com tais medidas pudéssemos fazer inferências mais seguras sobre os resultados da VFC. Além disso, o nível dos atletas analisados foi baixo (Yo Yo IR1 $=1272 \pm 340,9 \mathrm{~m}$ ) quando 
comparado com atletas de elite, impossibilitando, dessa forma, a extrapolação dos resultados da forte relação observada entre a VFC inicial e o $\Delta \%$ no Yo-Yo IR1 para atletas com melhor desempenho. Outra limitação foi o tamanho da amostra analisada. Essa limitação se deve ao fato de que a pesquisa realizada em modalidades coletivas se restringe ao número de atletas disponível no elenco da equipe avaliada a qual também está sujeita a perdas amostrais pelos seguintes fatores: os goleiros são retirados das análises, atletas que se lesionam e não terminam as avaliações, que são transferidos e que se apresentam após o início da pesquisa. Entretanto, o tamanho amostral do presente estudo está de acordo com diversos estudos que têm investigado esportes coletivos ${ }^{22-24}$.

Em que pesem algumas limitações do presente estudo, novas informações sobre a relação entre o sistema nervoso autônomo e desempenho foram produzidas. Os resultados sugerem que atletas com maior modulação autonômica são mais responsivos aos treinamentos. De fato, $72 \%$ da variação no Yo Yo IR1 pode ser explicada pelos índices vagais da VFC obtidos no momento pré-treinamento. Essas informações são importantes para técnicos e preparadores físicos criarem estratégias de individualização de cargas de treinamento e tentar minimizar a variabilidade interindividual dos atletas no que diz respeito à modulação autonômica e ao desempenho.

\section{CONCLUSÕES}

O presente estudo demonstrou que seis semanas de pré-temporada foram capazes de promover uma melhora do desempenho no Yo-Yo IR1 e na velocidade de $30 \mathrm{~m}$. A partir dos resultados do presente estudo é possível afirmar que atletas com maior modulação autonômica são mais responsivos aos treinamentos. Consequentemente, informações sobre o sistema nervoso autônomo dos atletas obtidas a partir a análise da VFC podem servir de parâmetro para os treinadores criarem estratégias de individualização de cargas de treinamento e tentar minimizar as diferenças interindividuais de modulação autonômica e desempenho.

\section{REFERÊNCIAS BIBLIOGRÁFICAS}

1. Vigne G, Gaudino C, Rogowski I, Alloatti G, Hautier C. Activity Profile in Elite Italian Soccer Team. Int J Sports Med 2010;31(5):304-10.

2. Mohr M, Krustrup P, Bangsbo J. Match performance of high-standard soccer players with special reference to development of fatigue. J Sports Sci 2003;21(7):519-28.

3. Lambert M, Borresen J. Measuring training load in sports. International journal of sports physiology and performance. Int J Sports Physiol Perform 2010;5(3):406-11.

4. Bangsbo J, Iaia F, Krustrup P. The Yo-Yo intermittent recovery test: A useful tool for evaluation of physical performance in intermittent sports. Sports Med 2008;38(1):37-51.

5. Krustrup P, Mohr M, Amstrup T, Rysgaard T, Johansen J, Steensberg A, et al. The yo-yo intermittent recovery test: physiological response, reliability, and validity. Med Sci Sports Exerc 2003;35(4):697-705.

6. Castagna C, Impellizzeri F, Cecchini E, Rampinini E, Alvarez JCB. Effects of intermittent-endurance fitness on match performance in young male soccer players. J Strength Cond Res 2009;23(7):1954-9. 
7. Rampinini E, Sassi A, Azzalin A, Castagna C, Menaspà P, Carlomagno D, et al. Physiological determinants of Yo-Yo intermittent recovery tests in male soccer players. Eur J Appl Physiol 2010;108(2):401-9.

8. Borresen J, Lambert MI. Autonomic control of heart rate during and after exercise: measurements and implications for monitoring training status. Sports Med 2008;38(8):633-46.

9. Manzi V, Castagna C, Padua E, Lombardo M, D’Ottavio S, Massaro M, et al. Dose-response relationship of autonomic nervous system responses to individualized training impulse in marathon runners. Am J Physiol Heart Circ Physiol 2009;296(6):H1733-40.

10. Iellamo F, Legramante JM, Pigozzi F, Spataro A, Norbiato G, Lucini D, et al. Conversion from vagal to sympathetic predominance with strenuous training in high-performance world class athletes. Circulation 2002;105(23):2719-24.

11. Kiviniemi AM, Hautala AJ, Kinnunen H, Tulppo MP. Endurance training guided individually by daily heart rate variability measurements. Eur J Appl Physiol 2007;101(6):743-51.

12. Hautala AJ, Mäkikallio TH, Kiviniemi A, Laukkanen RT, Nissilä S, Huikuri $\mathrm{HV}$, et al. Cardiovascular autonomic function correlates with the response to aerobic training in healthy sedentary subjects. Am J Physiol Heart Circ Physiol 2003;285(4):H1747-52.

13. Bangsbo J. Yo-yo test. Ancona, Italy: Kells. 1996.

14. Markovic G, Dizdar D, Jukic I, Cardinale M. Reliability and factorial validity of squat and countermovement jump tests. J Strength Cond Res 2004;18(3):551-5.

15. Gamelin FX, Berthoin S, Bosquet L. Validity of the polar S810 heart rate monitor to measure RR intervals at rest. Med Sci Sports Exerc 2006;38(5):887-93.

16. Hopkins WG, Marshall SW, Batterham AM, Hanin J. Progressive statistics for studies in sports medicine and exercise science. Med Sci Sports Exerc 2009;41(1):3-13.

17. Buchheit M, Chivot A, Parouty J, Mercier D, Al Haddad H, Laursen P, et al. Monitoring endurance running performance using cardiac parasympathetic function. Eur J Appl Physiol 2010;108(6):1153-67.

18. Hedelin R, Bjerle P, Henriksson-Larsén K. Heart rate variability in athletes: relationship with central and peripheral performance. Med Sci Sports Exerc 2001;33(8):1394-8.

19. Bangsbo J, Mohr M, Krustrup P. Physical and metabolic demands of training and match-play in the elite football player. J Sports Sci 2006;24(7):665-74.

20. Rampinini E, Bishop D, Marcora S, Ferrari Bravo D, Sassi R, Impellizzeri F. Validity of simple field tests as indicators of match-related physical performance in top-level professional soccer players. Int J Sports Med 2007;28(3):228-35.

21. Kiviniemi AM, Hautala AJ, Seppänen T, Mäkikallio TH, Huikuri HV, Tulppo MP. Saturation of high-frequency oscillations of RR intervals in healthy subjects and patients after acute myocardial infarction during ambulatory conditions. Am J Physiol Heart Circ Physiol 2004;287(5):H1921-7.

22. Milanez VF, Pedro RE, Moreira A, Boullosa DA, Salle-Neto F, Nakamura FY. The role of aerobic fitness on session rating of perceived exertion in futsal players. Int J Sports Physiol Perform 2011;6(3):358-66.

23. Milanez VF, Ramos SP, Salle-Neto F, Machado FA, Nakamura FY. Relação entre métodos de quantificação de cargas de treinamento baseados em percepção de esforço e frequência cardíaca em jogadores jovens de futsal. Rev Bras Educ Fís Esporte 2012;26(1):17-27.

24. Pedro RE, Milanez VF, Boullosa DA, Nakamura FY. Running speeds at ventilatory threshold and maximal oxygen consumption discriminate futsal competitive level. J Strength Cond Res 2012;28:[In press].
Endereço para correspondência

Fábio Yuzo Nakamura Universidade Estadual de Londrina, Centro de Educação Física e Esporte, Departamento de Educação Física Rod. Celso Garcia Cid, km 380, Campus Universitário

CEP: 86051-990 - Londrina, PR. Brasil E-mail:fabioy_nakamura@yahoo.com.br 